RECENT RESULTS ON POLARIZATION PHENOMENA

H. SpInka
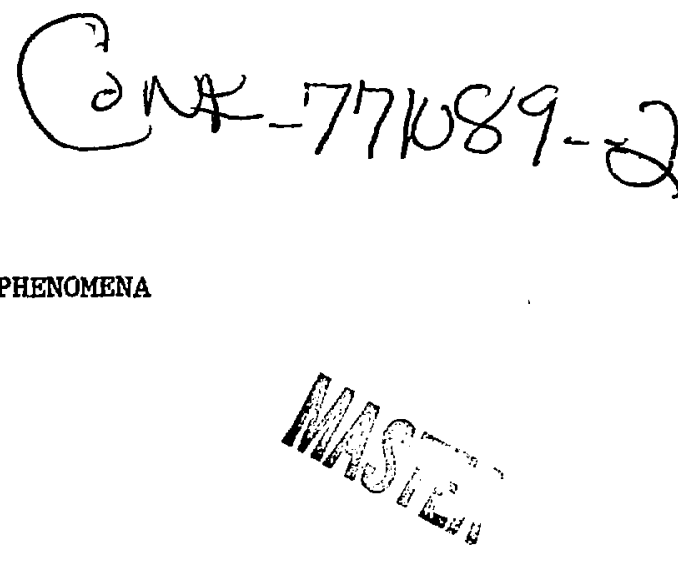

Prepared for

\author{
Arnual Meeting \\ Division of Particles \& Fields \\ Argonne National Laboratory \\ Argonne, IL \\ November 6-8, 1977
}


The facilities of Argonne National Laboratory are owned by the United States Government. Under the terms of a contract (W-31-109-Eng-38) between the U. S. Department of Energy, Argonne Universities Association and The University of Chicago, the University emplcys the staff and operates the Laboratory in accordance with policies and programs formulated, approved and reviewed by the Association.

\section{MEMBERS OF ARGONNE UNIVERSITIES ASSOCIATION}

The University of Arizona

Carnegie-Mellon University

Case Western Reserve University

The University of Chicago

University of Cincinnati

Illinois Institute of Technology

University of Illinois

Indiana University

Iowa State University

The University of Iowa
Kansas State University The University of Kansas Loyola University . Marquette University Michigan State University The University of Michigan University of Minnesota University of Missouri Northwestern University University of Notre Dame
The Ohio State University Ohio University

The Pennsylvania State University

Purdue University

Saint Louis University

Southern Illinois University

The University of Texas at Austin

Washington University

Wayne State University

The University of Wisconsin

\section{NOTICE}

This report was prepared as an account of work sponsored by the United States Government. Neither the United States nor the United States Department of Energy, nor any of their employees, nor any of their contractors, subcontractors, or their employees, makes any warranty, express or implied, or assumes any legal liability or responsibility for the accuracy, completeness or usefulness of any information, apparatus, product or process disclosed, or represents that its use would not infringe privately-owned rights. Mention of commercial products, their manufacturers, or their suppliers in this publication does not imply or connote approval or disapproval of the product by Argonne National Laboratory or the U. S. Department of Energy. 


\title{
RECENT RESULTS ON POLARIZATION PHENOMENA
}

\author{
H. Spinka \\ Argonne National Laboratory, Argonne, Il. 60439 \\ INTRODUCTION
}

Today I would like to discuss several topics relating to polarization phenomena. First, the structure iri certain Pp spin observables measured at Argonne will be described, and a number of new results presented. These data may indicate the existence of a dibaryon resonance. Next, there are some recent results from the Indiena group on high energy inclusive proton polarization from Fermilab. The values are sizeable and they will be compared to the inclusive $\Lambda$ polarization data at high energies. Finally, there are some new measurements of pp elastic scattering polarization at high energy from CERN and Fermilab. Some general features of these data will be aiscussed. Polarization phenomena at large $\mathrm{P}_{\mathrm{T}}{ }^{2}$ will be covered in the talk by A. Krisch and will not be reviewed here.

\section{A. IOW ENERGY PP DATA}

There are some new results on pp spin parameters from several experimental groups at Argonne. I would like to concentrate on the significance of the structure observed in the total cross-section difference for longitudinally-polarized beam and target.

First, consider the helicity amplitudes used to describe the experimental data. There are three nonzero amplitudes at $t=0$, namely $\phi_{1}, \phi_{2}$ and $\phi_{3}:$

$$
\begin{aligned}
& \phi_{1}=\langle++\mid++\rangle \\
& \phi_{2}=\langle-\mid++\rangle \\
& \phi_{3}=\langle t-\mid+-\rangle
\end{aligned}
$$

and there are three total cross sections which have been measured,

$$
\begin{aligned}
& \sigma_{\text {Tot }}=\frac{2 \pi}{k_{\text {cm }}} \operatorname{Im}\left\{\phi_{1}(0)+\phi_{3}(0)\right\}=\frac{1}{2}\left\{\sigma_{\text {Tot }}(\vec{t})+\sigma_{\text {Tot }}(\ddagger)\right\} \\
& \Delta \sigma_{I}=\frac{4 \pi}{k_{\mathrm{cm}}} \operatorname{Im}\left\{\phi_{1}(0)-\phi_{3}(0)\right\}=\sigma_{\text {Tot }}(\vec{t})-\sigma_{\text {Tot }}(\vec{f}) \\
& \Delta \sigma_{T}=-\frac{4 \pi}{k_{c m}} \operatorname{Im}\left\{\phi_{2}(0)\right\} \quad=\sigma_{T o t}(t+)-\sigma_{T o t}(t+)
\end{aligned}
$$

*Fork supported by the 0.S. Energy Research and Development Administration. 
where the arrows denote the bean and target spin directions in the laboratory frame. The first is the ordinary spin-averaged cross section $\sigma_{\text {Tot }}$. The other two are cross-section differences for longitudinal $\left(\Delta \sigma_{I}\right)$ and transverse $\left(\Delta \sigma_{T}\right)$ protons.

The data are shown in Fig. 1. The open triangles and open circles correspond to preliminary regults. All cross-section measurements $\left(\sigma_{T O E}, \Delta \sigma_{I}, \Delta \sigma_{T}\right)$ were performed as standard transmission experiments. The data are all plotted on the same scale to show that spin effects are quite sizeable at these energies. The $\Delta \sigma_{T}$ measurements were performed by two groups; one was a Michigan, Argonne, St. Iouis collaboration ${ }^{1}$ and the other was a Rice, Michigan, Houston group ${ }^{2}$. There appears to be some sort of structure near $2 \mathrm{GeV} / \mathrm{c}$. Additional measurements are planned to clarify the variation of $\Delta \sigma_{T}$ with energy.

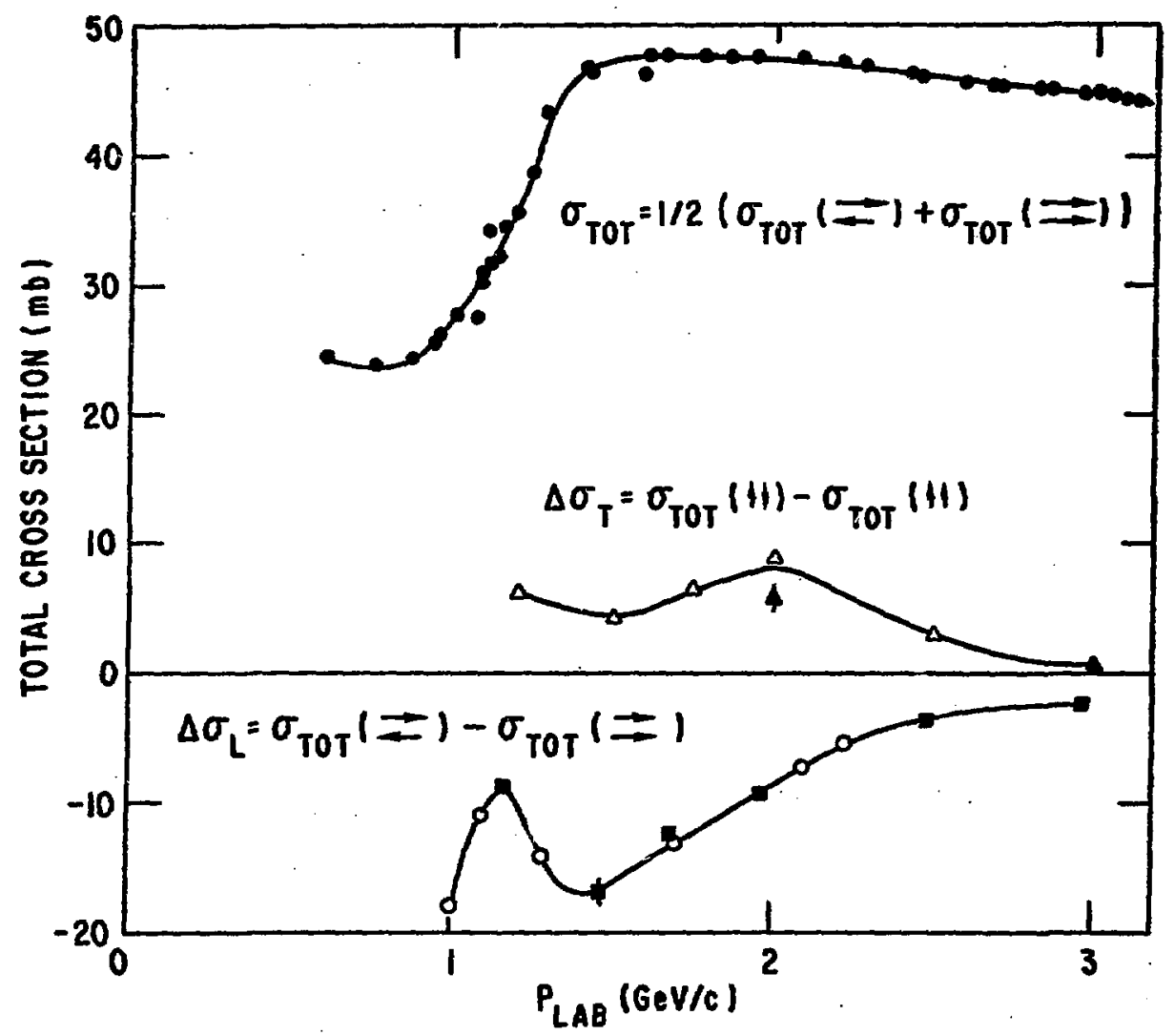

Fig. 1 Total cross sections for pp interactions. The upper curve is the spin-averaged cross section, the middle curve is the crosssection difference for transversely-polarized beam and target, and the lower curve is the total cross-section difference for longitudinally-polarized beam and target. The open triangles ${ }^{2}$ and open circles 5 are preliminary results. The curves are drawn to guide the eye. 
The $\Delta \sigma_{L}$ data, measured by the Argonne in-house polarization group. ${ }^{3-5}$ exhibit rather striking structure as a function of energx. Combining the $\Delta \sigma_{I}$ and total cross-section data, then the imaginary parts of the helicity amplitudies $\phi_{1}$ and $\phi_{3}$ can be separated,

$$
\begin{aligned}
& \text { In } \phi_{1}(0)=\frac{k_{\mathrm{cm}}}{4 \pi}\left(\sigma_{\text {Tot }}+\Delta \sigma_{I} / 2\right) \\
& \operatorname{Im} \phi_{3}(0)=\frac{k_{\mathrm{cm}}}{4 \pi}\left(\sigma_{\text {Tot }}-\Delta \sigma_{I} / 2\right) .
\end{aligned}
$$

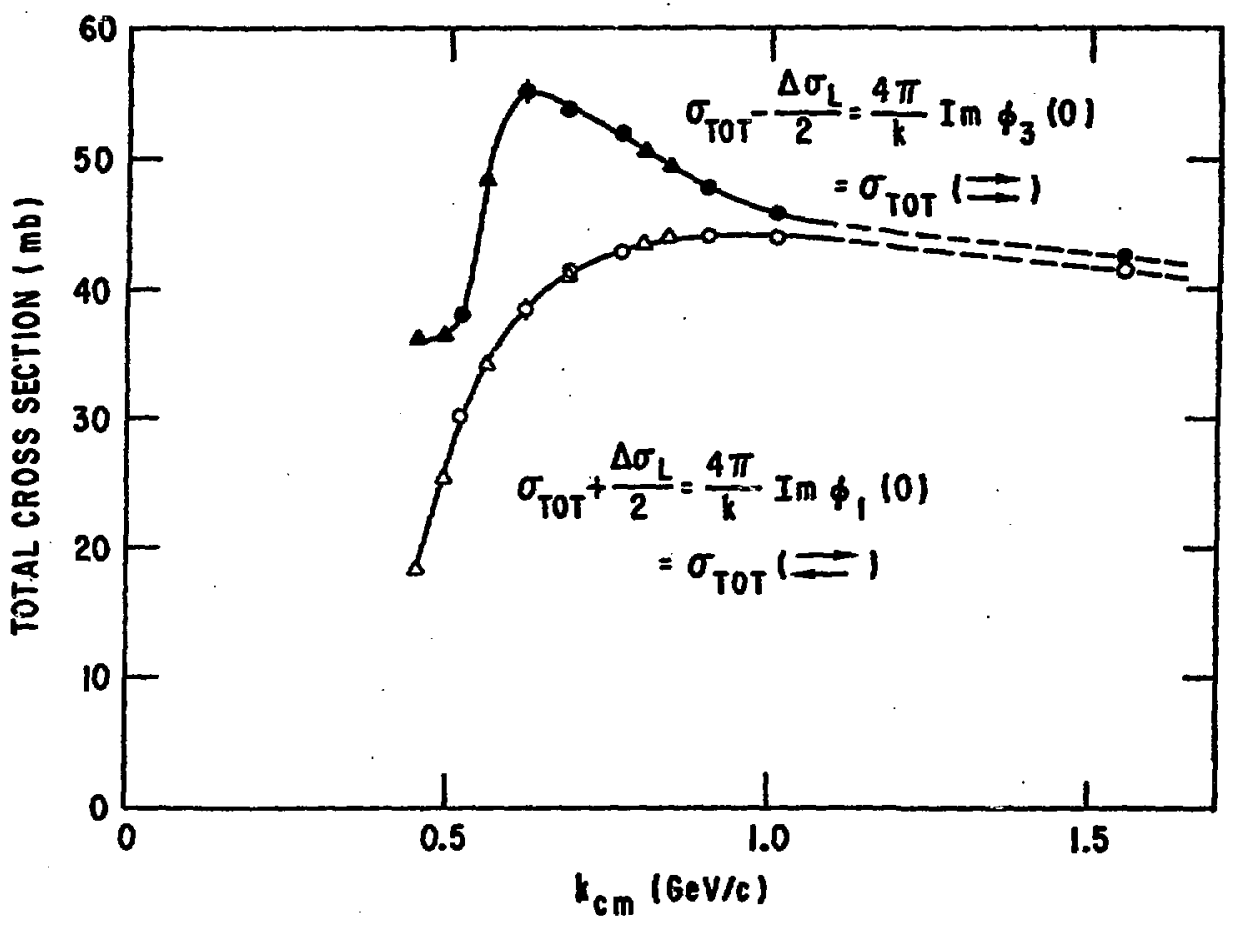

Fig. 2 Total cross sections for pure longitudinal spin states. The triangles correspond to preliminary data and the circles to data from references 3 and 4 . Note that the structure appears only in $\phi_{3}(0)$. 
The results are shown in Fig. 2. The spin-averaged cross section is the average of these two curves. It can be seen that the structure is present in $\phi_{3}$ but not in $\phi_{1}$.

Decomposing the helicity amplitudes into partial waves, then

$$
\begin{aligned}
& \phi_{1}=\text { singlet }+ \text { triplet }(J=I \pm I \text {, even }) \\
& \phi_{2}=- \text { singlet }+ \text { triplet }(J=I \pm 1 \text {, even }) \\
& \phi_{3}=\quad \text { triplet' }(J=I \pm 1 \text {, even })+ \\
& \text { triplet }(J=I \text {, oda) , }
\end{aligned}
$$

where singlet refers to spin-singlet contributions and triplet to spin-triplet contributions. The fact that the structure in $\Delta \sigma_{r}$ appears in $\phi_{3}$ but not in $\phi_{1}$ indicates that the structure is cautsed by spin-triplet, $J=I=$ odd partial waves.

Grein and $\mathrm{Kroll}^{6}$ have applied fixed-t dispersion relations to the $\Delta \sigma_{L}$ results. In terms of the amplitude $A_{3}$,

$$
A_{3}=\frac{P_{1 \mathrm{ab}}}{k_{\mathrm{cm}}}\left(\phi_{1}-\phi_{3}\right)
$$

then

$$
\operatorname{Im}\left(A_{3}\right)=\frac{P_{1 a b}}{4 \pi} \Delta \sigma_{L} .
$$

Grein and Kroll used phase-shift results for $\Delta \sigma_{I}$ ăt $t_{4}$ low energies, the measured values of $\Delta \sigma_{\text {o }}$ between 1 and $6 \mathrm{Gev} / \mathrm{C}, 3,4$ and some assumptions about the high energy behavior of $\Delta \sigma_{\text {, }}$, to obtain the imaginary part of $A_{3}$ as a function of energy as shown in Fig. 3. Then the real part of $\mathrm{A}_{3}$ was computed from dispersion relations. (This is similar to the calculation of the real part of the forward scattering amplitude from the total cross section ( $\sigma_{\text {Tot }}$ ) measurenents in the spin-averaged case).

The amplitude $A_{3}$ is displayed in an Argand diagram in Fig. 4. Since the structure in $\Delta \sigma_{F}$ and $A_{3}$ arises from the helicity amplitude $\phi_{3}$, and since $A_{3} \sim\left(\Phi_{1}-\phi_{3}\right)$, the amplitude $\left(-A_{3}\right)$ is actually plotted. It exhibits a clear resonance-like behavior corresponding to a dibaryon resonance at $P_{1 a b}=1.5 \mathrm{GeV} / \mathrm{c}$ in the $p p$ system. 


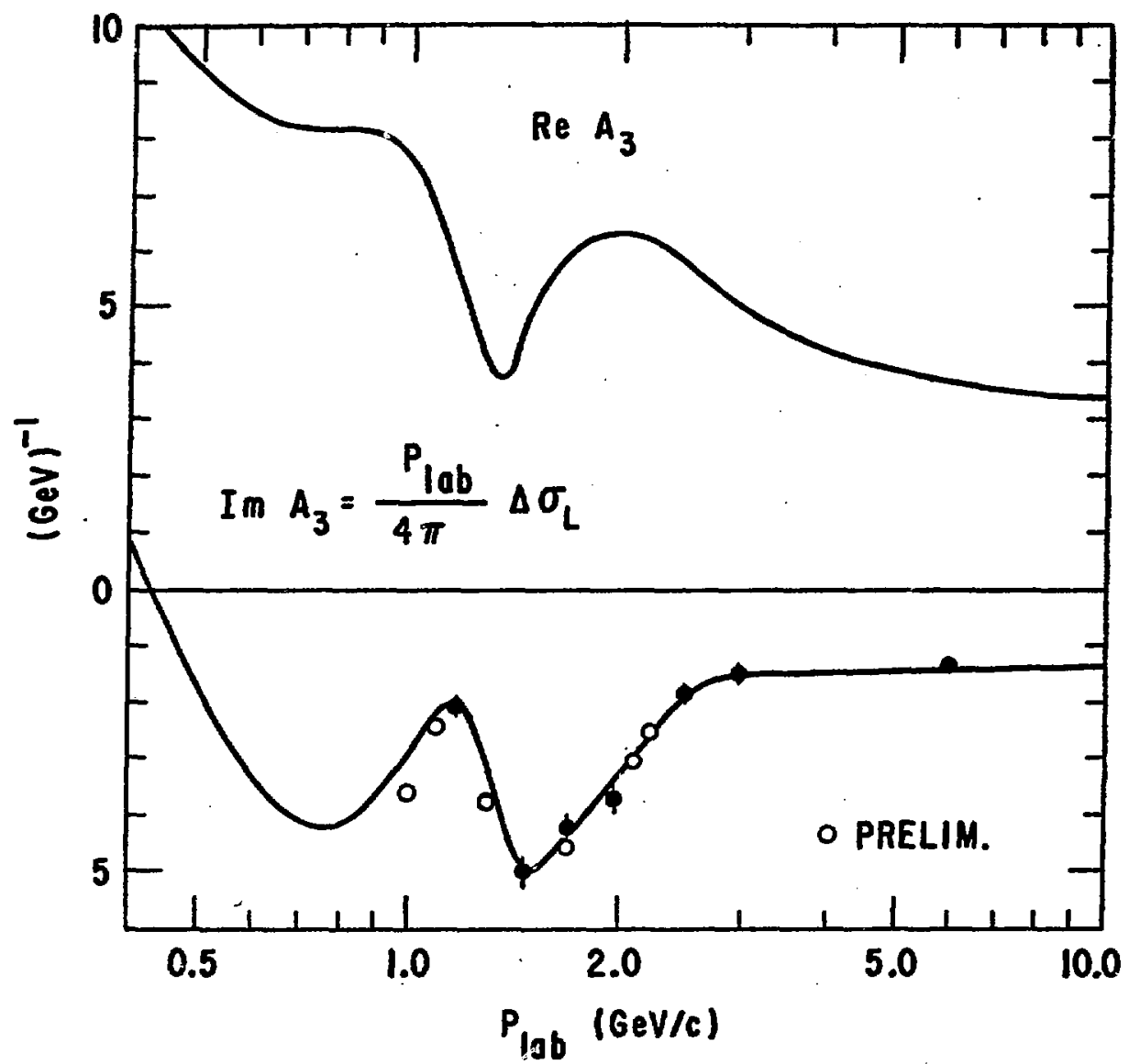

Pig. 3 The real and imaginary parts of the amplitude $A_{3}$ from Grein and Kroll. 6 The imaginary part was obtained from phase-shift results below $1 \mathrm{GeV} / \mathrm{c}$ and from $\Delta \sigma_{\mathrm{L}}$ data (filled circles) between 1 and $6 \mathrm{GeV} / \mathrm{c}$. For comparison, the preliminary $\Delta \sigma_{L}$ values (open circles) are also shown. The real part of $A_{3}$ was calculated from the imaginaxy part of $\mathrm{A}_{3}$ using dispersion relations. 


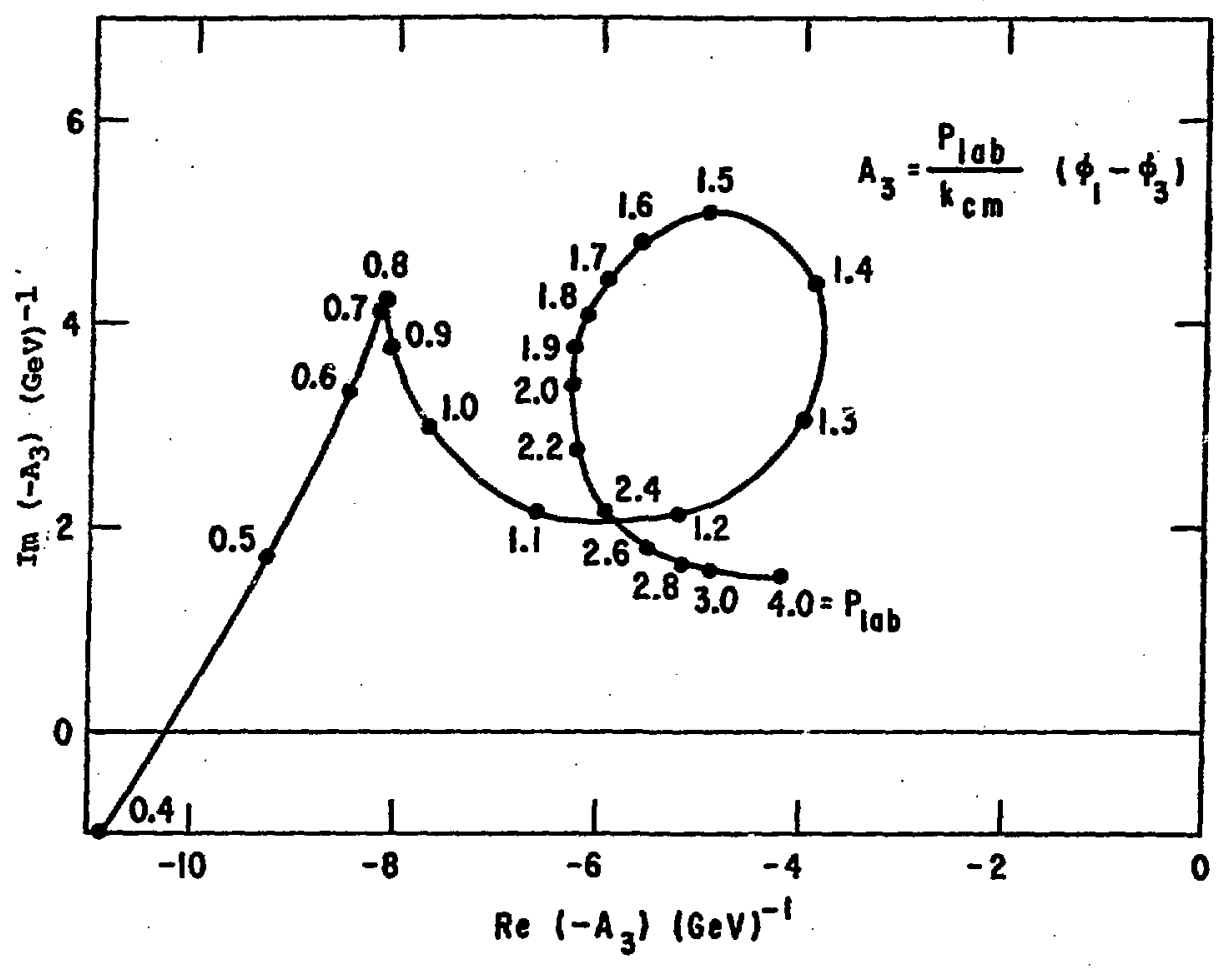

Fig. 4 Argand diagram of the amplitude $\left(-a_{3}\right)-\left(\phi_{3}-\phi_{1}\right)$ from reference 6 . Tr 3 structure in $\Delta \sigma_{I}$ corresponas to resonance-like behariox in $A_{3}$ at $P_{1 a b} \simeq 1.5 \mathrm{Gev} / \mathrm{C}$.

$\star * * * * * * * * * * * * * * * * * * *$

Additional evidence for this dibaryon resonance comes from a number of pp elastic scattering measurements. The total elastic cross section, shown in Fig. 5, seems to exhibit a peak at about $1.5 \mathrm{GeV} / \mathrm{c}$, though some improvement in these data would be desixable. 


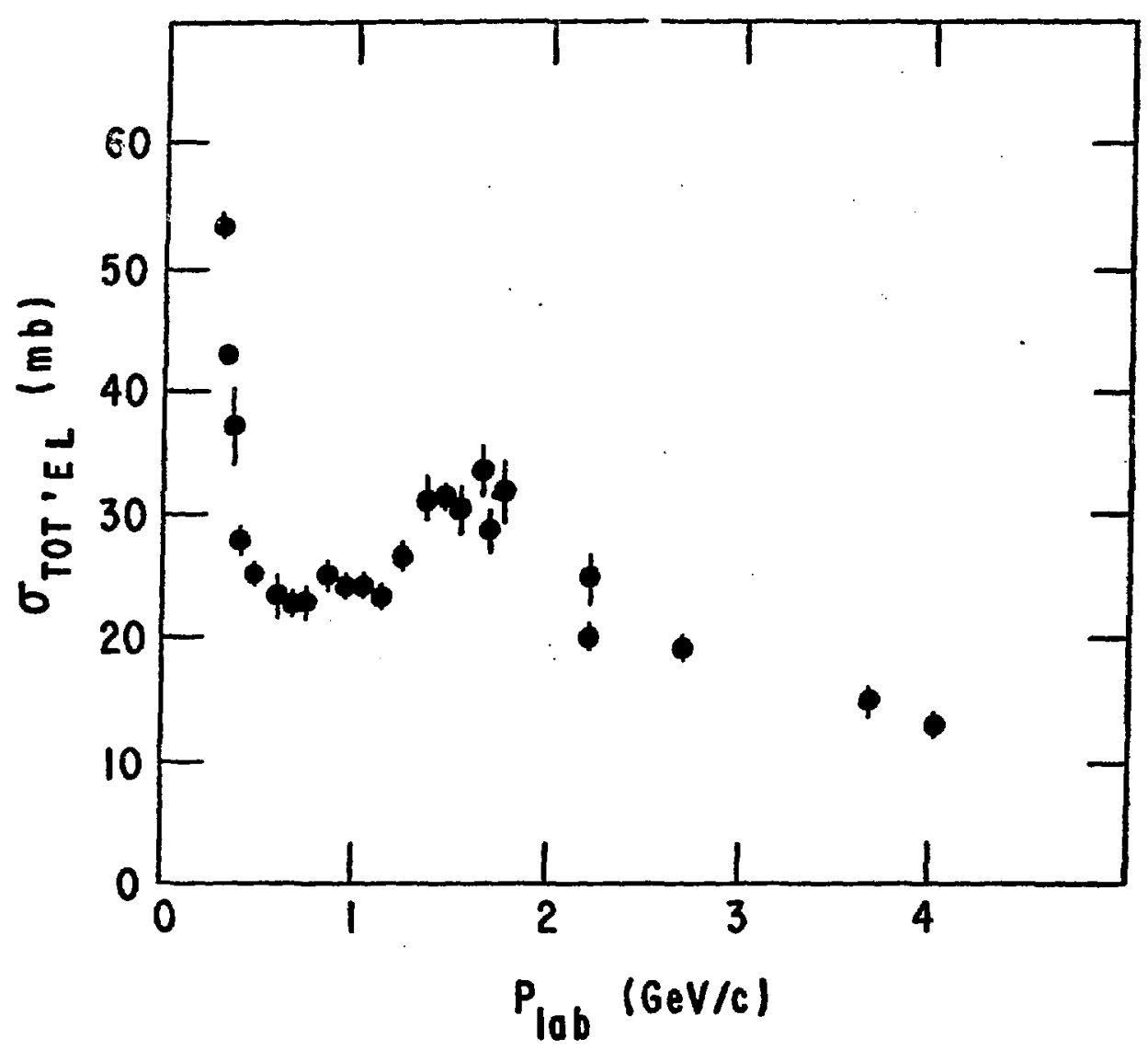

Fig. 5 Total cross section for pp elastic scattering.

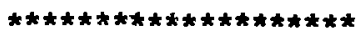

Next, consider two elastic scattering spin-spin correlation measurements at $\theta_{\mathrm{cm}}=90^{\circ}$. The first, $C_{\mathrm{NNN}}\left(90^{\circ}\right)$, has both the beam and the target polarized transverse to the scattexing plane. The other; $C_{\text {IL }}\left(90^{\circ}\right)$, has both polarized longitudinally. The resuits are shown in Fig. 6 . Over the past 20 years, many groups have measured $C_{\mathrm{NN}}\left(90^{\circ}\right)$, as indicated by the numerous points at momenta below $2.5 \mathrm{GeV} / \mathrm{c}$. These were either double or triple scattering experiments, and consequently often of poor statistical accuracy. with the combination of a polarized beam and a polarized 
target, higher precision measurements are possible. The statistical accuracy of some recent data from Argonne by the Michigan group 7 is quite good, as indicated in Fig. 7. They plan measurements of $C_{\text {NN }}\left(90^{\circ}\right)$ up to $12 \mathrm{GeV} / \mathrm{c}$, where their present $C_{\mathrm{NN}}(\theta)$ results seem to be 0.2 to 0.3 and increasing with $\theta$ at the largest angles measured so far. Additional high-precision $C_{\text {NN }}\left(90^{\circ}\right)$ measurements in the range $P_{1 a b} \sim 1$ to $2 \mathrm{GeV} / \mathrm{c}$ would be quite helpful.

The $C_{\text {LI }}\left(90^{\circ}\right)$ data in Fig. 6 are preliminary. Values at additional energies will be available soon. (The $\Delta \sigma_{I}$ and $C_{I I I}\left(90^{\circ}\right.$ ) measurements were performed simultaneously by the Argonne in-hous: polarization group.)

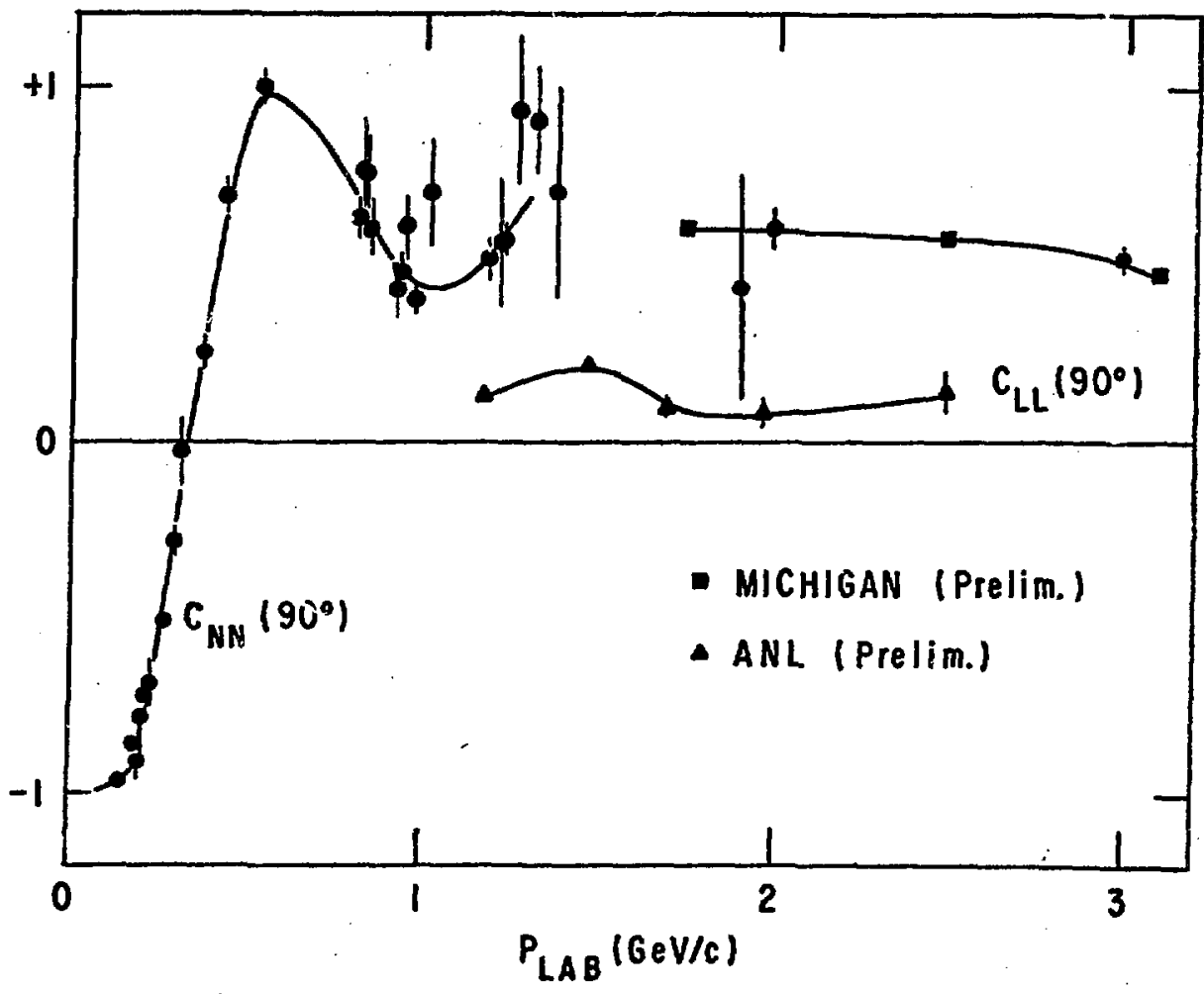

Fig. 6 The spin-spin correlation parameters $C_{\mathrm{NN}}$ and $C_{\mathrm{IL}}$ at. $\theta_{\mathrm{cm}}=$ 90\%. The solid squares and triangles are preliminary data from references 5 and 7 . 


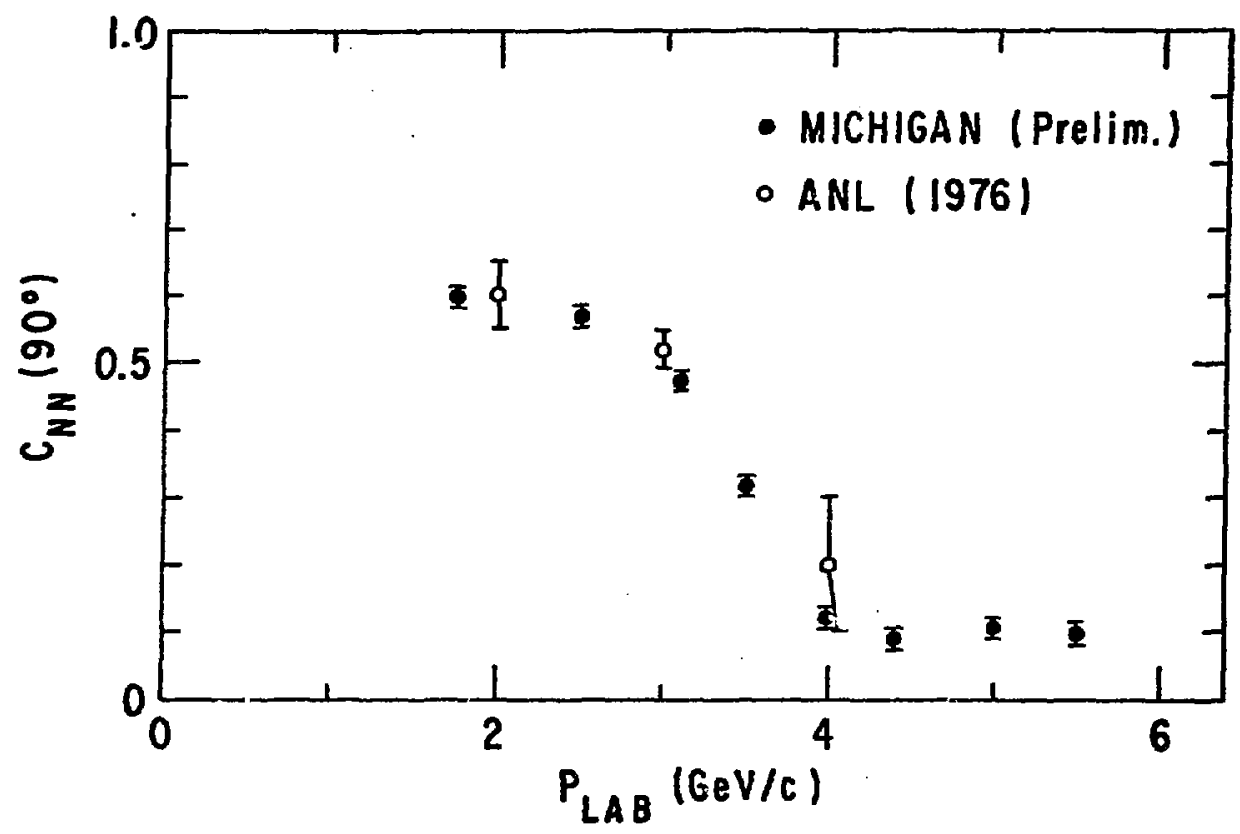

Fig. 7 The spin-spin correlation parameter $\mathrm{C}_{\mathrm{NN}}\left(90^{\circ}\right)$ as measured at Argonne. 7, 6

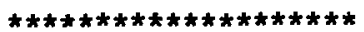

The value of $\mathrm{C}_{\mathrm{NN}}\left(90^{\circ}\right)$ is given by the spin-triplet cross section at $90^{\circ}$ minus the spin-singlet cross section, divided by the sum. The expression for $C_{\text {LL }}\left(90^{\circ}\right)$ is similar except that the spin-triplet term in the numerator is an interference of various triplet contributions:

$$
\begin{aligned}
& c_{\text {WN }}\left(90^{\circ}\right)=\frac{\text { triplet }- \text { singlet }}{\text { triplet }+ \text { singlet }} \\
& c_{\text {LI }}\left(90^{\circ}\right)=\frac{\text { triplet (mixture) - singlet }}{\text { triplet }+ \text { singlet }} .
\end{aligned}
$$


There is a peak in $\mathrm{C}_{\text {I }}\left(90^{\circ}\right)$ and an indication of a peak in $c_{\text {. }}\left(90^{\circ}\right.$ ) near $1.5 \mathrm{GE} / \mathrm{C}$ seen in Fig. 6. Therefore, the spintriplet contribution is sizeable in both the total cross sections and in the elastic channel at $\theta_{\mathrm{sm}}=90^{\circ}$.

One final bit of evidence from the elastic channel comes from the polarization parameter measured as a function of energy, shown in Fig. 8. The values have been averaged over the t-range of 0.1 to 0.2 , and all data shown were collected by one group in order to minimize systematic differences between the points. The polarization times the differential cross section contains only spin-triplet terms, and it shows a significant peak near $1.5 \mathrm{GeV} / \mathrm{c}$. Therefore, measurements of $\mathrm{C}_{\mathrm{NN}}\left(90^{\circ}\right), \mathrm{C}_{\mathrm{fI}}\left(90^{\circ}\right)$ and polarization in $\mathrm{pp}$ elastic scattering are $\mathrm{all}$ consistent $\mathrm{w}^{*}+\mathrm{h}$ structure in the spin-triplet partial waves $n \in: r 1.5 \mathrm{GeV} / \mathrm{c}$.

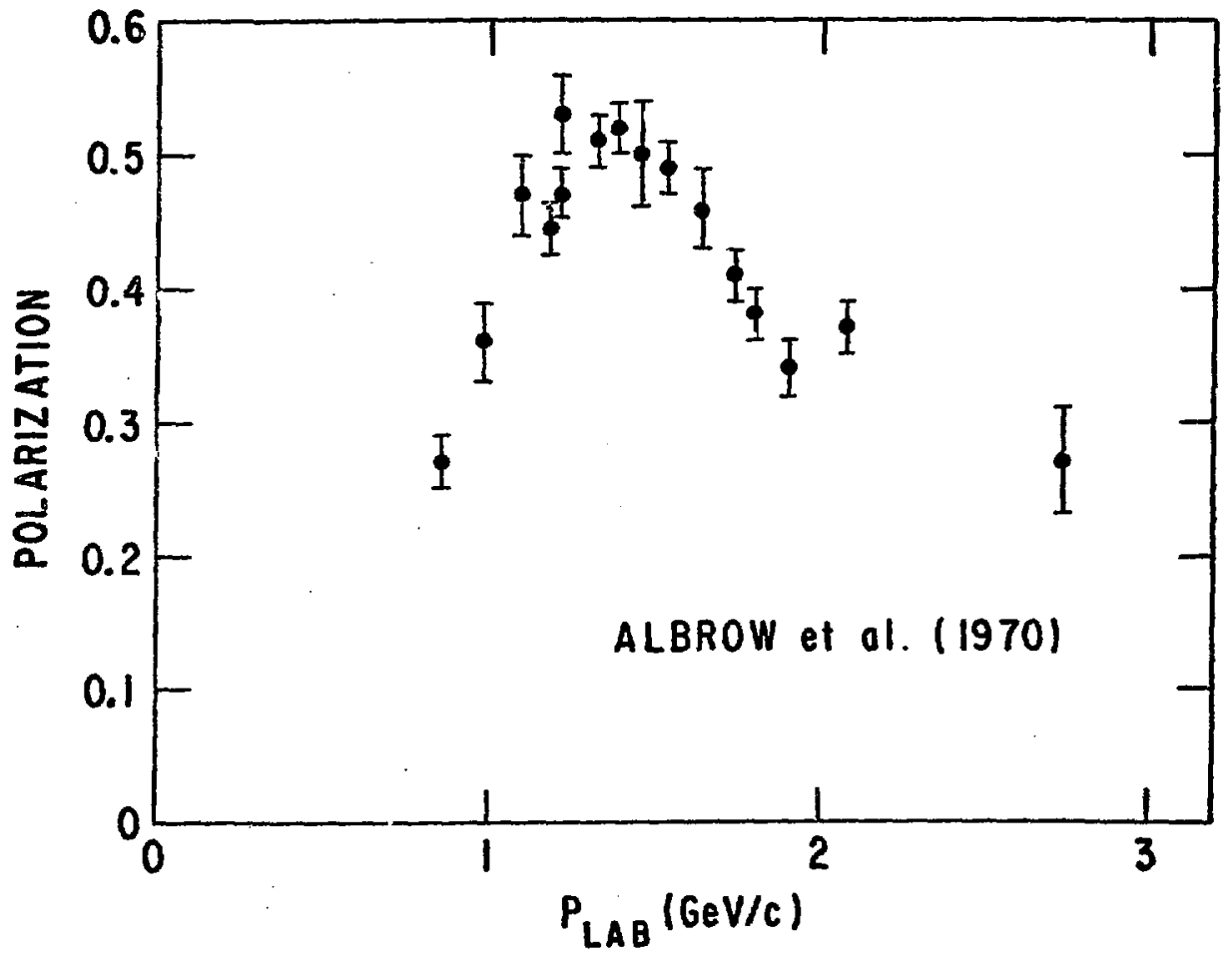

Fig. 8. The polarization parameter for $p$ p elastic scattering averaged over $0.1 \leq|t| \leq 0.2(\mathrm{Gev} / \mathrm{c})^{2}$ from reference 9 . 
A Legendre coefficient analysis was performed by Hidaka et al. at Argonnelo The elastic angular distribution and the polarization as a function of angle are fit by the expressions:

$$
\begin{aligned}
\frac{d \sigma}{d \Omega} & =\frac{i}{k^{2}} \quad \sum A_{N} P_{N}(\cos \theta) \\
P \frac{d \sigma}{d \Omega} & =\frac{1}{k^{2}} \quad \sum B_{N} P_{N}^{1}(\cos \theta) .
\end{aligned}
$$

The coefficients $\mathrm{H}$ and $\mathrm{B}_{\mathrm{N}}$ are rolated to the individual partial waves. The variation of the coefficients as a function of energy indicates that the partial wave ${ }^{3} F_{3}$ is responsible for the structure seen in the polarization. A phase-shift analysis performed by Hoshizakili comes to the same conclusion. Note that the ${ }_{F_{3}}$ partial wave is consistent with the $J=I=$ odd condition derived from the observation of strusture in $\phi_{3}(0)$ but not in $\phi_{1}(0)$.

Therefore, assuming the structure corresponds to a resonance, then its properties would be

$$
\begin{array}{lc}
\text { Mass } & -2250 \mathrm{MeV} \\
\text { Width } & \sim 200 \mathrm{MeV} \\
\text { Charge } & 2 \\
\mathrm{~J}^{\mathrm{P}} & 3^{-}
\end{array}
$$

A dibaryon with these quantum numbers has not been predicted to my knowledge. In order to fit such a resonance into the six quark bag model of Jaffe, ${ }^{12}$ where all quarks are in an S-state, orbital excitation of the quarks would have to be included.

An argument could also te made that the structure observed in $\Delta \sigma_{T}$ ne: $: 2 \mathrm{GeV} / \mathrm{c}$ might be a resonance. However, at this time the evidence is much weaker than for the structure near $1.5 \mathrm{GeV} / \mathrm{c}$ in $\Delta \sigma_{\mathrm{L}}$. For example, the structure in $\Delta \sigma_{\mathrm{T}}$ probably corresponds to spin-singlet partial waves. In this case, the numerous pp polarization data give no information since only spin-triplet partial waves contribute.

\section{B. HIGH-ENERGY INCLUSIVE POLARIZATION DATA}

The Indiana Universit: Group has recently performed an experiment at Fermilab to measure inclusive proton polarizations: Accelerating protons in the Fermilab main ring struck an internal carbon target. The inclusively-produced protons were detected in a superconducting spectrometer at $\theta_{1 \text { ab }}=52^{\circ}$. which was set to record particles with $\mathrm{P}_{\mathrm{T}}=1.1 \mathrm{GeV} / \mathrm{c}$. The proton polarization was measured in a carbon polarimeter, and pions were rejected by time 
of flight requirements. The missing masses detected ranged from $M^{2}=30$ to $100 \mathrm{GeV}^{2}$ at $100 \mathrm{GeV}$ and from 80 to $140 \mathrm{GeV}^{2}$ at $400-\mathrm{GeV}$ incident protons, and $x=0.89$.

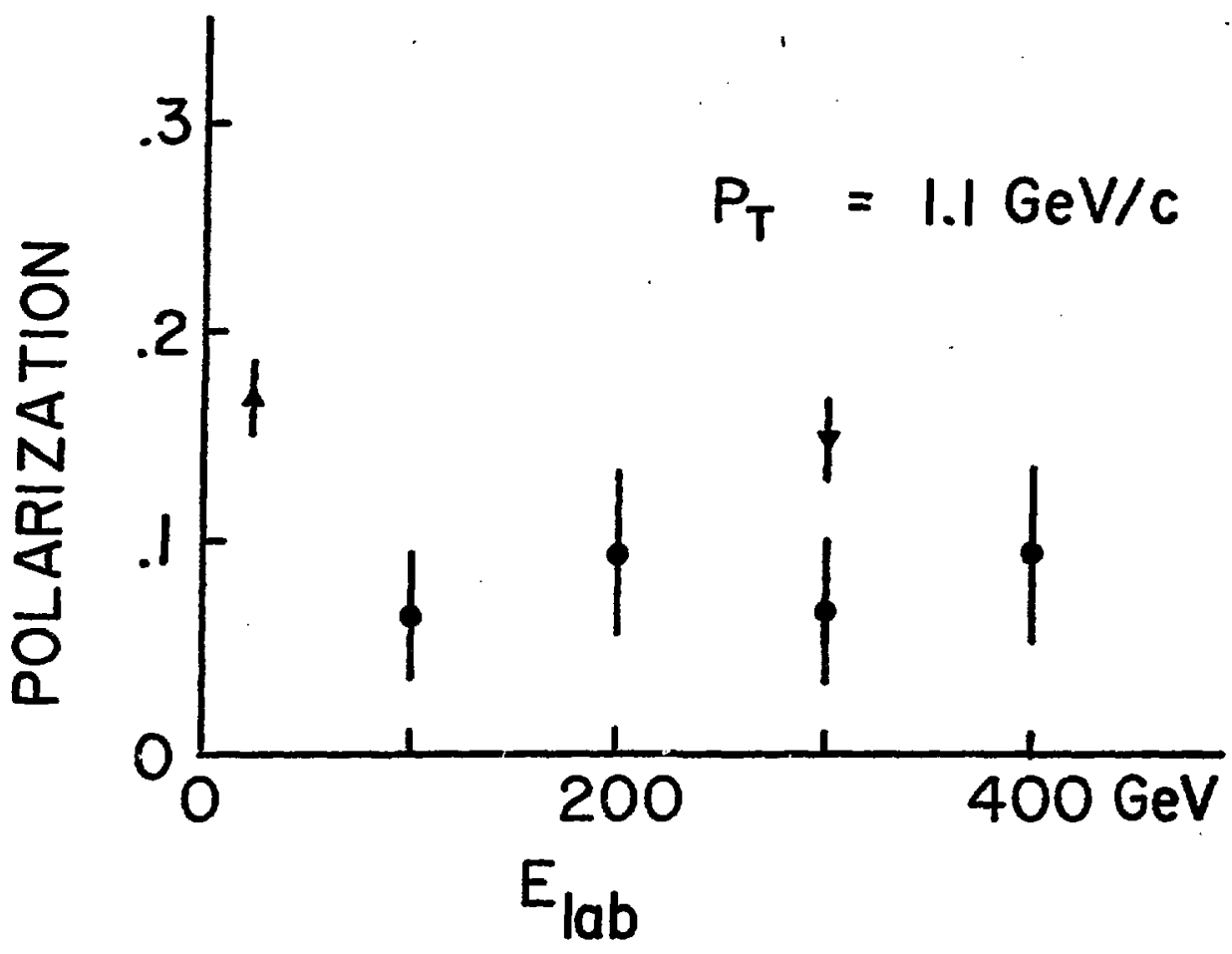

Fig. 9 Inclusive polarization data at high energies and $P_{T}=1.1$ GeV/c. The circles are preliminary inclusive proton data for $p+c$ interactions, ${ }^{13}$ and the triangles are inclusive $\Lambda$ polarizations for $\mathrm{p}+\mathrm{Be}\left(\boldsymbol{V}\right.$, reference 14 ) and for $\mathrm{p}+\mathrm{Pt}\left(\boldsymbol{A}_{\text {, reference }}\right.$ i5) interactions.

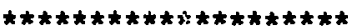

The results are shown in Fig. 9. The sizeable polarizations (6 - 108) are essentially independent of energy within the error bars, and they are much larger in magnitude than expected from any rimple Regge model. These inclusive-proton polarizations are reminiscent of the large $\Lambda$ polarizations seen in another experiment at Fermilab. The Wisconsin, Michigan, Rutgers' Group 14,15 measured a large inclusive $\Lambda$ polarization at $300 \mathrm{GeV}$ which seems independent 
of energy down to $24 \mathrm{GeV} / \mathrm{C}$ at $P_{T} \geq 1.0 \mathrm{GeV} / \mathrm{c}$, as shown in Fig. 9. If the $\Lambda$ and proton inclusive polarizations are related, there may be an increase in the inclusive proton polarization at higher $P_{T}$, which is seen in the $\Lambda$ case. The Indiana grcup plans to investigate this possibility in the near future.

\section{HIGH ENERGY PP POLARIZATION RESULIS}

There are several new measurements of the polarization parameter in pp elastic scattering at high energy (see Figs. 10, Il, and 12). The CERN data at $24 \mathrm{GeV} / \mathrm{c}^{16}$ and the S: pukhov data at $45 \mathrm{GeV} / \mathrm{c}^{17}$ have both been rublished, except for the high-t points at $24 \mathrm{GeV} / \mathrm{c}$. In addition, there are some other results at these energies recently obtained using a hydrogen jet target at Fermilab by the Indiana group. 18 They measured the polarization of the recoil proton as a function of energy at $-t=0.3,0.6,0.8$ and 1.0 $\mathrm{GeV} / \mathrm{C})^{2}$. Their data agree well with the results from the other groups who used polarized-proton targets. The Fermilab E-61 collaboration of Argonne, Berkeley, Fermilab, harvard, Suffolk and Yale obtained polarization data at 100 and $300 \mathrm{Gev} / \mathrm{c}^{19,20}$ with a double-arm magnetic spectrometer. A similar experimental setup was used at the CERN-SPS by the CERN, Padova, Trieste, vienna collaboration to measure the polarization at $150 \mathrm{GeV} / \mathrm{c} .21$

These results have several features in common. The absolute value of the polarization is quite small (less than .05) out to $-t=0.8(\mathrm{GeV} / \mathrm{c})^{2}$ at all energies shown in Figs. 10 and 11 . The polarization is positive at small-t and seems consistently negative at $-t=1.0$. The polarization data at 45 and $150 \mathrm{GeV} / \mathrm{c}$ are indistinguishable. However, the t-dependence of the 100 and $300 \mathrm{GeV} / \mathrm{c}$ results beyond $-t=0.5$ is somewhat different from the $45 \mathrm{GeV} / \mathrm{c}$ data. The differences are probably within the quoted statistical uncertainties, though a sizeable energy dependence of the data at $|t|>0.5(\mathrm{Gev} / \mathrm{c})^{2}$ is not excluded. Finally, at $|t|>1$ there may be rapid changes in the polarization with $t$ and with maximum asymetries of \pm .20 .

Predictions from two Regge models are given on the plots of the 100 and 300-Gev/c data in Figs. 10 and 1l. The solid curves are from the model of Field and stevens ${ }^{22}$ with a pure non-flip Pomeron. Their 100-GeV/c curve is somewhat high at small- $t$, and their $300 \mathrm{GeV} / \mathrm{c}$-prediction agrees fairly well with the data. The dashed curve is from a model of Pumplin and Kane. 23 .Their model has a spin-flip component of the Pomeron, which leads to polarizations on the order of -0.10 even at ISR energies. Figvie 12 shows the data plotted at fixed-t as a function of energy. The PumplinKane prediction is roughly -0.10 at $-t \simeq 1(\mathrm{GeV} / \mathrm{c})^{2}$, which agrees with the high-energy measurements. Their pre'sction at $t=0.3$ is shown in Fig. 12, and the data are consistently smaller than the theoretical value at most energies shown. 


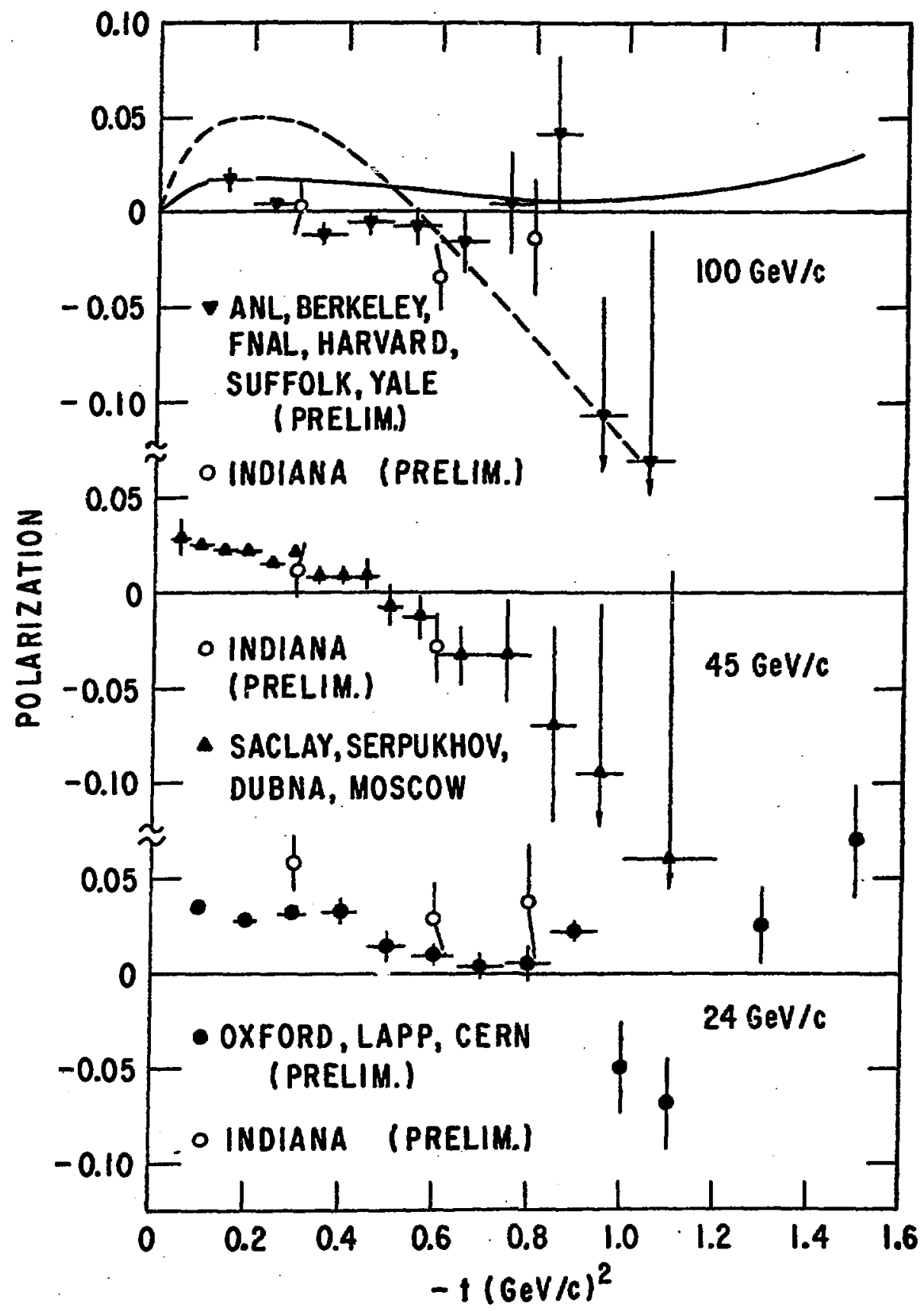

Fig. 10 Polarization for pp elastic scattering at 24, 45 and $100 \mathrm{GeV} / \mathrm{c}$. 


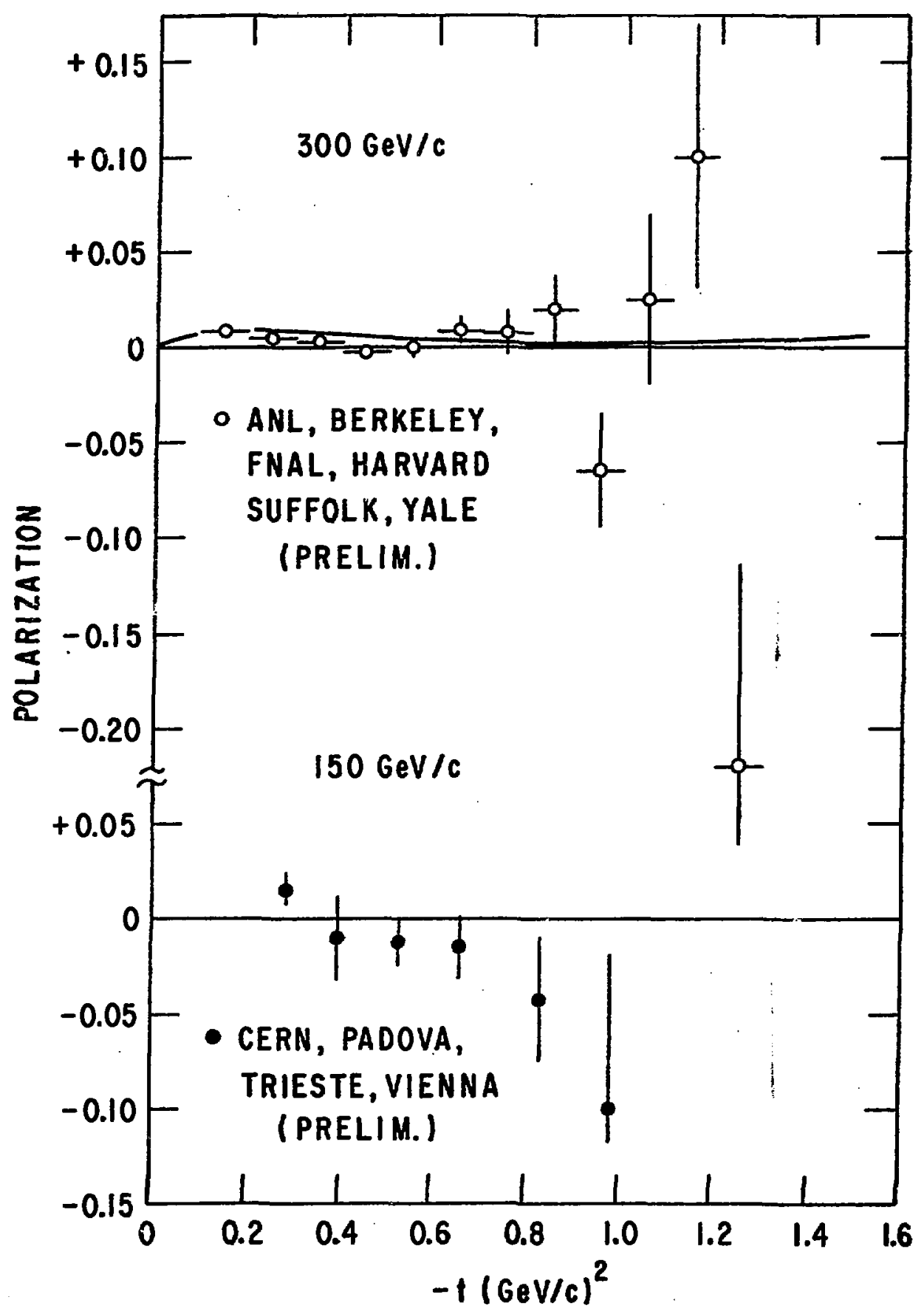

Fig. 11 Polarization for pp elastic scattering at 150 and $300 \mathrm{GeV} / \mathrm{c}$. 


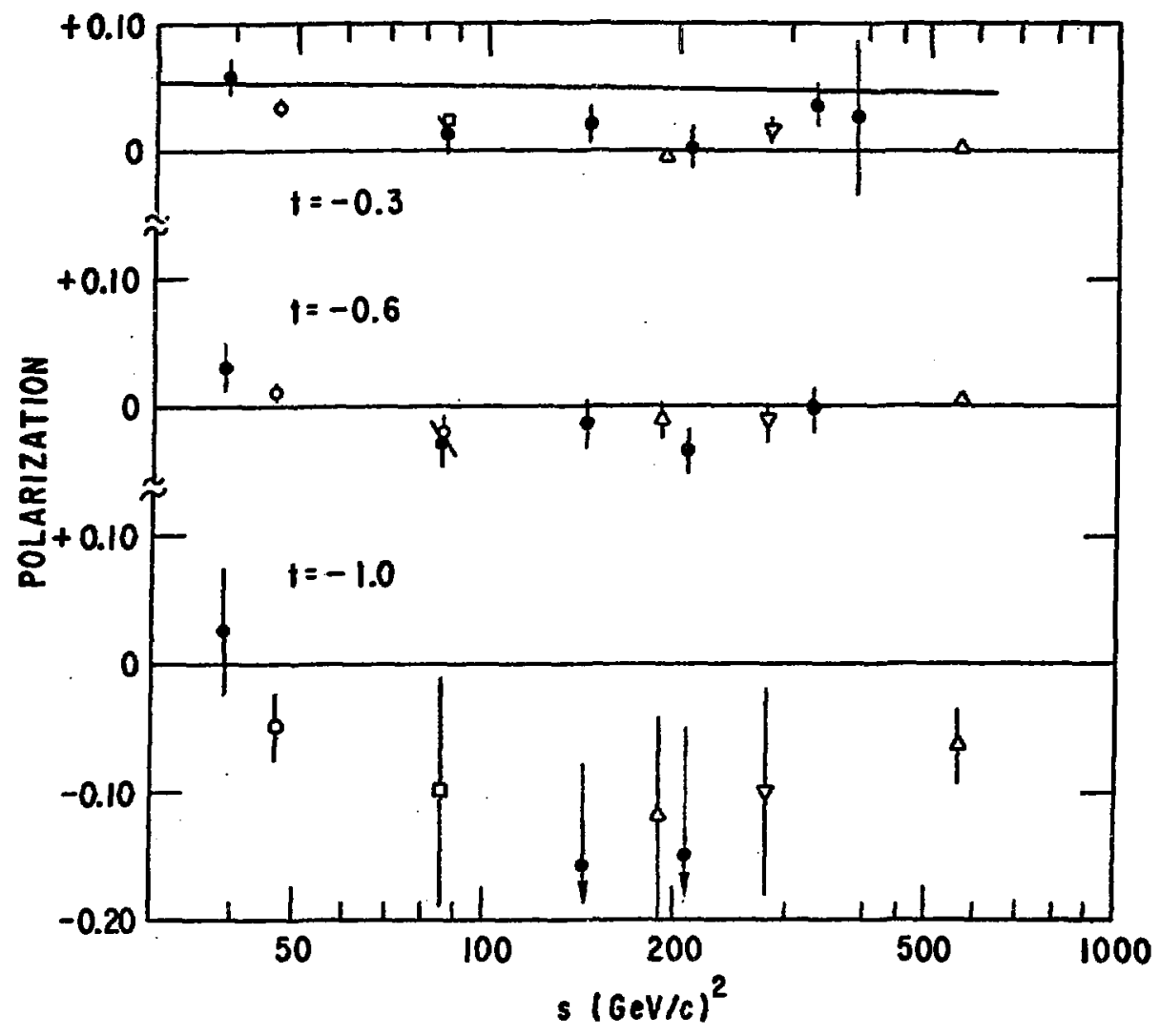

Fig. 12 Polarization in pp elastic scattering at fixed-t. The measurements are from the Indiana group (6, Reference 18), CERN (0, Reference 16), Serpukhov (D, Reference 17), the Fermilab E-61 collaboration $(\Delta$, Reference 19 , and 20 ) and the CERN-SPS group $(\nabla$, Reference 21). The prediction of the Pumplin-Kane model at $-t=0.3$ is also shown. 
Polarization effects at largex $-t$ will be studied by the Fermilab E-бl collaboration and the CERN-SPS group. We can look forward to quite interesting results from these experiments, especially in the region of the dip in the pp elastic cross section, where there are already hints of sizeable structure in the polarization at $300 \mathrm{Gev} / \mathrm{c}$.

\section{REFERENCES}

1. W. DeBoer et al., Phys. Rev. Lett., 34, 558 (1975).

2. J.B. Roberts, private comnunication; $\bar{E}$. Biegert et al., to be published.

3. I.P. Auer et al., Phys. Lett., 67B, 113 (1977).

4. I.P. Auer et al., Phys. Lett., 70B, 475 (1977).

5. I.P. Auer et al., to be published.

6. W. Grein and P. Kroll, University of Wuppertal preprint WU B 77-6; P. Kroll, private communication.

7. A. Krisch, private communication.

8. D. Miller et al., Phys. Rev. Lett., 36, 763 (1976); D. Miller et al., to be published in Phys. Rev., D16.

9. M.G. Albrow et al., Nucl. Phys., B23, $44 \overline{5(1970) .}$

10. K. Hidaka et al., Phys. Lett., 70B, 479 (1977).

11. N. Hoshizaki, Prog. Theor. Phys., 58, 716 (1977).

12. R.I. Jaffe, Phys. Rev. Iett., 38, 195 (1977).

13. S. Gray, private communication; M. Corcoran et al.. Indiana University preprint IUHEE \#10.

14. G. Bunce et al., Phys. Rev. Lett., 36, 1113 (1976).

15. K. Heller et al., Phys. Lett., 68B, 480 (1977).

16. D.G. Crabb et al., Nucl. Phys., B121, 231 (1977).

17. A. Gaidot et al., Phys. Lett., 61B, 103 (1976).

18. S. Gray, private communication; M. Corcoran et al., Indiana University preprint IUHEE \#9.

19. I.P. Auer et al., Phys. Rev. Lett., 39, 313 (1977).

20. A. Yokosawa, private communication.

21. G. Fidecaro et al., contribution to the European Conference on Particle Physics, Budapest, Hungary, 4-9 July, 1977.

22. R. Field, private communication.

23. J. Pumplin and G.L. Kane, Phys. Rev., Dll, 1183 (1975). 